\title{
Controlled Optofluidic Crystallization of Colloids Tethered at Interfaces
}

\author{
Alessio Caciagli, ${ }^{1}$ Rajesh Singh $\odot,{ }^{2}$ Darshana Joshi, ${ }^{1}$ R. Adhikari $\odot,{ }^{2,3}$ and Erika Eiser $\oplus^{1, *}$ \\ ${ }^{1}$ Cavendish Laboratory, 19 JJ Thomson Avenue, Cambridge CB3 OHE, United Kingdom \\ ${ }^{2}$ DAMTP, Centre for Mathematical Sciences, University of Cambridge, Wilberforce Road, Cambridge CB3 OWA, United Kingdom \\ ${ }^{3}$ The Institute of Mathematical Sciences-HBNI, CIT Campus, Chennai 600113, India
}

(Received 17 March 2020; accepted 8 July 2020; published 5 August 2020)

\begin{abstract}
We report experiments that show rapid crystallization of colloids tethered to an oil-water interface in response to laser illumination. This light-induced transition is due to a combination of long-ranged thermophoretic pumping and local optical binding. We show that the flow-induced force on the colloids can be described as the gradient of a potential. The nonequilibrium steady state due to local heating thus admits an effective equilibrium description. The optofluidic manipulation explored in this work opens novel ways to manipulate and assemble colloidal particles.
\end{abstract}

DOI: 10.1103/PhysRevLett.125.068001

Since their introduction [1], optical tweezers have revolutionized the manipulation of matter at the nano- to micrometer scale [2-6]. Tweezers have found extensive use in the trapping and assembly of micron-sized colloidal particles [7-9] and have enabled the formation of novel forms of colloidal matter that are held together by optical forces [10-16]. While the various mechanisms of optical trapping are well understood, much less is known about the effects of light-induced heating on the flow of the surrounding fluid medium, in particular near a liquid-liquid interface. Naively, one might think that, at a liquid-liquid interface, the fluid motion would be dominated by Marangoni flow, which should drive the fluid away from the hot spot $[17,18]$. However, in our experiments, surface tethered colloids moved toward the hot spot, even when they are out of range of the direct optical binding forces [19].

In this Letter, we show that the optical trapping of a single colloidal particle near a water-oil interface can set up a long-ranged, nonequilibrium force field, which causes colloidal particles that are tethered to the surface but otherwise freely diffusing, to move to the hot spot where they crystallize. The sign, magnitude, and distance dependence of this nonequilibrium force cannot be accounted for by static colloidal interactions nor by surface-tension driven Marangoni flows. Using theory and simulation, we show that the experimentally observed colloidal motion is produced by stalled thermophoresis of a single, optically trapped colloid. In fact, to a good approximation, the stalled particle acts as a hydrodynamic monopole. For motion parallel to the interface, the hydrodynamic drag force behaves as the gradient of a potential. As a consequence, the particle dynamics admits an effective equilibrium description, allowing us to perform Brownian dynamics simulations. As we show below, the results of these simulations are in excellent agreement with experiments.
Importantly, the effective attractive potential depends only on the thermophoretic mobility of the stalled colloid. It manifests itself in simple solvents such as water or oil and is of much longer range than the optical trapping potential. As a consequence, interfacially trapped colloids can be used as switchable pumps. Such addressable pumps would enable novel strategies for optofluidic manipulation and the controlled assembly of colloidal particles [20]. Below, we describe our experimental results, theoretical analyses, and numerical simulations.

Sample geometry.-The inner panels of Fig. 1 show a sketch of our sample geometry. Oil droplets with a radius between 20 and $30 \mu \mathrm{m}$ were coated with a surfactantpolymer layer, following the protocol described in Ref. [21]. Onto this layer, we grafted a dense brush of single-stranded DNA sequences (denoted by $A$ ). Polystyrene particles (PS) of radius $a=0.53 \mu \mathrm{m}$, functionalized by complementary $A^{\prime}$ single-stranded DNA strands, were then allowed to hybridize with the $A$ chains on the surface. The DNA coating of the colloids prevents them from aggregating. As the colloids are tethered to the surface, rather than embedded in it, they are not subject to capillary forces, light-wave reflections, or long-ranged electrostatic dipolar interactions that would be caused by the asymmetry of charge distributions on interfacially wetted colloids [22,23]. Moreover, the colloids do not deform the surfactant-polymer coating, as the DNA tethering keeps the colloids some $50 \mathrm{~nm}$ away from the surface layer. As the oil droplets are much larger than the colloids, the interface is effectively flat on the scale probed in our experiments. Although tethered to surface polymers, the colloids are otherwise free to diffuse along the interface.

When a laser beam is focused above the oil-water surface, it will trap a single tethered colloid. This colloid will act as the thermophoretic "pump" that will recruit other tethered colloids. However, a fraction of the colloids remain 

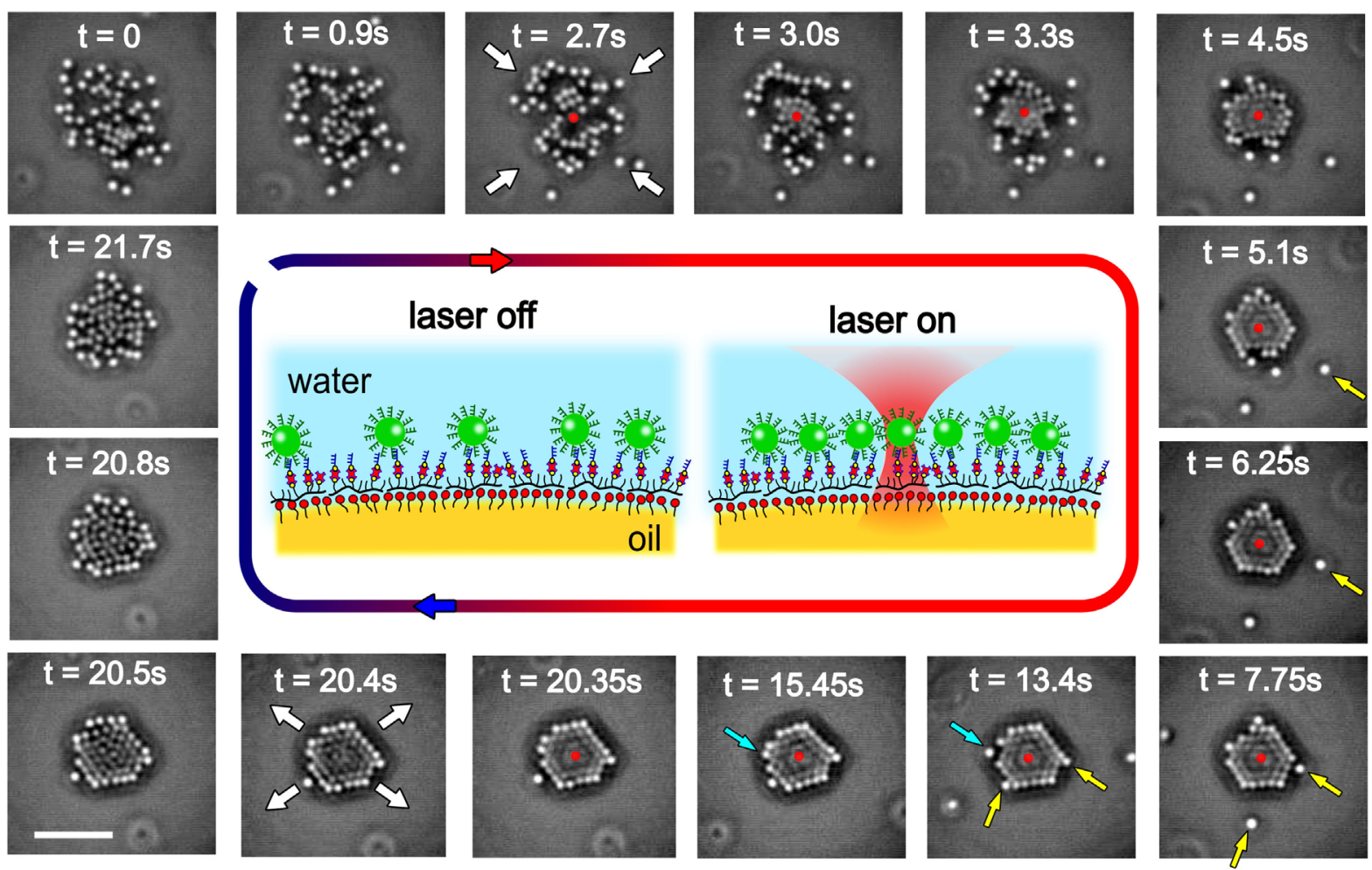

\begin{tabular}{|c|c|c|c|c|c|}
\hline 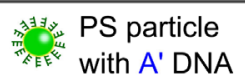 & Ifree $_{\text {PLL-PEG }}^{\text {biotinylated }}$ & $\circ$ biotin & $\sigma$ & streptavidin & $\begin{array}{l}\hbar_{0} \text { biotinylated } \\
\text { DNA A }\end{array}$ \\
\hline
\end{tabular}

FIG. 1. The panels in the rim of the figure show a time trace (clockwise) of the video-microscopy images of the light-induced entrapment and release of $0.53 \mu \mathrm{m}$ large polystyrene colloids tethered to the water-oil interface (the sale bar is $20 \mu \mathrm{m}$ ). At $t=2.7 \mathrm{~s}$, the laser is switched on (red arrow), trapping a single colloid (red dot; the width of the dot indicates the approximate width of the laser focus). Subsequently, optophoretic pumping brings other colloids to the hot spot, where they form a crystal stabilized by optical binding. At $t=20.4 \mathrm{~s}$, the laser is switched off (blue arrow), and the crystal dissolves. The motion of the particles from yellow and cyan arrows provides clear evidence of a long-ranged attraction to the center. The two central panels give a schematic side view of the water-oil interface coated with anionic surfactant [sodium dodecyl sulphate (SDS)] onto which a cationic block-copolymer [biotinylated poly- $L$ lysine (PLL)-polyethylene glycol (PEG)] is adsorbed. The biotinylated PEG ends are functionalized by streptavidin [21], which in turn binds to a single-stranded DNA sequence (denoted by $A$ ). This $A$ DNA strand then hybridizes with complementary $\left(A^{\prime}\right)$ strands on the polystyrene (PS) colloids The keys at the bottom mark each component. The particles diffuse freely on the interface when the laser is off (left panel); a single particle is optically trapped when the laser is turned on (right panel). For details, see movie SV in the Supplemental Material [24].

untethered and diffuse freely in the bulk. These particles serve as tracers of the bulk fluid flow. Further details of the system and the calibration of the trap are provided in the Supplemental Material [24].

Reversible crystallization. - The outer panels of Fig. 1 show our principal experimental result. Following the frames clockwise starting from the top left, the pictures show the crystallization and dissolution of a colloidal crystal, as the laser is switched on (red arrow) and then switched off (blue arrow). The primary optically trapped colloid is shown in red. The first two frames show free diffusion when the laser is off. When the laser is turned on in the third frame, diffusion is immediately replaced by directed motion toward the trapped particle with speeds of up to $50 \mu \mathrm{m} / \mathrm{s}$. This leads to rapid crystallization which is essentially complete within a few seconds, as shown in the frame at $6.2 \mathrm{~s}$. Thermal fluctuations cause small displacements in the core of the crystallite but large ones at the edges where particle rearrangements take place, as shown in the frames between 6.2 and $20.3 \mathrm{~s}$. The crystallite begins to melt as soon as the laser is turned off and a freely diffusing state is recovered within a few seconds. This cycle of freezing and melting in response to turning the laser on and off is rapid, robust, and reproducible.

Optofluidic mechanism.-What force underlies this phenomenon? By examining the motion of the colloids as the laser is turned on, it is clear that the force has a range of at least $5 \mu \mathrm{m}$ and a magnitude of the order of $\mathrm{pN}$, directed radially inward to the trapped particle. At such distances, neither the direct optical trapping, nor the optical binding forces to be discussed below, can play a role. The entrainment of colloids by Marangoni flow can be 
immediately ruled out, as such a flow must point outward from the hot region surrounding the laser focus. Direct thermophoresis toward the hot colloid is also incompatible with the experimental data, as untethered colloids are seen to move first toward the heated colloid and then to move vertically away [Fig. 3(b)]: thermophoresis would result in isotropic attraction. In the absence of other plausible mechanisms, we are led to postulate the following: the colloid nearest to the laser focus is optically trapped and local heating near an interface between two fluids with different thermal conductivities induces an asymmetric thermal gradient in the surrounding fluid. This gradient pushes the colloid toward the interface, where it stalls (as its Soret coefficient is positive [46]). From that moment on, the thermal gradient along its surface drives a thermo-osmotic flow originating in a thin boundary layer around the colloid [36,47-49]. Since the colloid remains stalled, the thermoosmotic flow continues unabated, but produces no particle motion. This leads to a monopolar hydrodynamic counterflow in the fluid, with the monopole pointing normal to the interface and into the water phase. The long-ranged and attractive character of the flow entrains untrapped particles and draws them toward the focus. If the entrained colloids are tethered, they aggregate into crystallites under the action of the optofluidic force. The local crystalline order is enhanced by short-ranged forces, including those due to optical binding (see below). In contrast, untethered tracer colloids first move along the surface toward the trapped colloid, but then they are advected away from the surface. Such behavior is illustrated in Fig. 3(b) where an untethered particle appears and then disappears from the focal plane, consistent with the flow pattern shown in Fig. 2(b).

To make the above hypothesis quantitative and testable, we solve the equations of mass, momentum, and energy conservation in the fluid with appropriate boundary conditions at the colloid surfaces and the oil-water interface (detailed in [24]). The geometry is shown schematically in Fig. 2(a). We use the boundary integral representation for the momentum (Stokes) and energy (Laplace) equations to impose boundary conditions at the colloid surfaces and use appropriate Green's functions to satisfy the boundary conditions at the oil-water interface [38]. The integral equations are solved in a basis of irreducible tensorial harmonics to yield the temperature field $T$ and fluid flow velocity $\mathbf{v}$ in an externally imposed temperature field $T^{\infty}$ (representing laser heating). These are shown in Fig. 2(b) for a single trapped colloid. From these we obtain the thermophoretic force $\mathbf{F}^{T}$ on the trapped colloid and the optofluidic force $\mathbf{F}^{H}$ with which free colloids are attracted to the trapped colloid as

$$
\mathbf{F}^{T}=-\left.\frac{\mu_{T}}{\mu_{\perp}} \nabla T^{\infty}\right|_{1}, \quad \mathbf{F}^{H}=\left.\frac{\mu_{T}}{\mu_{\perp} \mu_{\|}} \mathbf{G}^{w} \cdot \nabla T^{\infty}\right|_{1} .
$$

In the above, $\mu_{T}$ is the thermophoretic mobility, $\mu_{\perp}$ and $\mu_{\|}$ are, respectively, the mobility perpendicular and parallel to
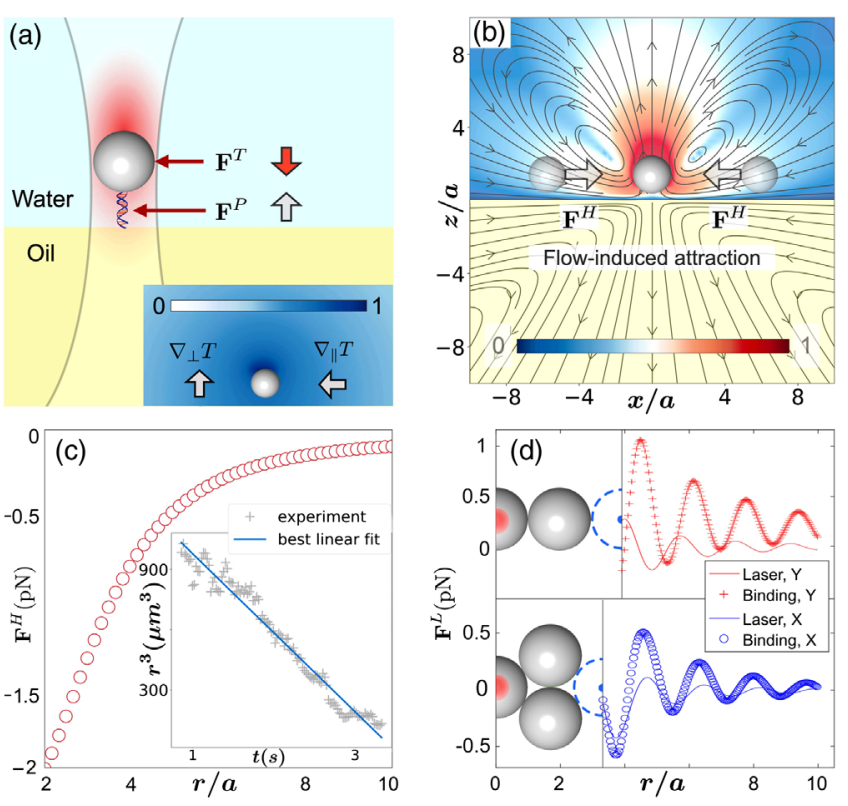

FIG. 2. Long-ranged optofluidic and short-ranged optical binding forces. (a) schematic of a trapped DNA-tethered colloid. The thermophoretic force $\mathbf{F}^{T}$ drives the colloid toward the interface compressing the tether and generating a reaction force $\mathbf{F}^{P}$. Motion stalls when $\mathbf{F}^{T}+\mathbf{F}^{P}=0$. The inset, false color plot of the temperature, shows gradients perpendicular $(\perp)$ and parallel $(\|)$ to the interface. (b) flow streamlines in the oil and water phases due to the stalled colloid which, to leading order, is a monopole of strength $-\mathbf{F}^{T}$. The color map is the logarithm of the speed of flow in the water phase normalized by the maximum. The flow entrains tethered untrapped particles leading to a optofluidic force $\mathbf{F}^{H}$. (c) Inverse-square variation of the magnitude of $\mathbf{F}^{H}$ with distance $r$ from the stalled colloid. The inset shows experimental $r(t)$ data for aggregating particles, following a $r^{3} \sim t$ scaling. (d) The variation of optical binding forces $\mathbf{F}^{L}$ with normalized $r / a$ parallel and perpendicular to the polarization of the trapping laser. Solid and broken lines are contributions from the trapping laser and the particle scattering forces, respectively. The local minima of $\mathbf{F}^{L}$ promote crystalline order.

the interface, $\mathbf{G}^{w}$ is a Green's function of the Stokes equation for a no-shear plane interface, and $\left.\right|_{1}$ indicates evaluation at the center of the trapped colloid [24]. The optofluidic force, through its dependence on $\mathbf{G}^{w}$, varies monotonically as the inverse square of the distance $r$ from the trapped colloid. As particle motion is overdamped, the velocity scales as $v \sim r^{-2}$, and hence displacements scale with time intervals as $r^{3}(0)-r^{3}(t) \sim t$. We test this from the experimentally measured positions to find excellent agreement, shown in the inset of Fig. 2(c).

Optofluidic potential.-For motion at a constant height, the nonequilibrium optofluidic force $\mathbf{F}^{H}=F^{H} \hat{\mathbf{r}}$ admits a potential

$$
\Phi(r)=-\frac{\mu_{T}}{4 \pi \eta \mu_{\perp} \mu_{\|}}\left[\frac{1}{1+\lambda} \frac{h}{r^{*}}+\frac{2 \lambda}{1+\lambda} \frac{h^{3}}{r^{*^{3}}}\right] \partial_{z} T^{\infty} .
$$


Here, $r^{*}=\sqrt{r^{2}+h^{2}}, h$ is the height of the colloid from the interface, and $\lambda=\eta_{o} / \eta_{w}$ is the ratio of the viscosities of oil and water. The optofluidic potential depends linearly on the ratio $\kappa_{o} / \kappa_{w}$ of thermal conductivity of the oil and water layers through the dependence on the temperature gradient [24]. Then, the in-plane coordinate $\mathbf{R}_{i}=\left(X_{i}, Y_{i}\right)$ of an untrapped colloid $(i=2,3, \ldots, N)$ obeys the overdamped Langevin equation

$$
d \mathbf{R}_{i}=-\mu_{\|} \nabla_{i}(U+\Phi) d t+\sqrt{2 k_{B} T \mu_{\|}} d \xi_{i},
$$

where $U$ is a potential containing the sum of all short-ranged colloid-tether and colloid-colloid interactions, $\Phi$ is the optofluidic potential evaluated at the location of the particle, and $d \boldsymbol{\xi}_{i}$ is a zero-mean Gaussian random variable with variance $\left\langle d \boldsymbol{\xi}_{i} d \boldsymbol{\xi}_{j}\right\rangle=\delta_{i j} \boldsymbol{I} d t$. The stationary distribution of the particle positions is Gibbsian, $P \sim \exp \left[-(U+\Phi) / k_{B} T\right]$, even though the dynamics is out of equilibrium. At an airwater interface, where $\lambda=0$, the optofluidic potential has a Coulomb form $\Phi \sim 1 / r^{*}$. The opposite limit of $\lambda \rightarrow \infty$, corresponding to a no-slip wall, gives an optofluidic potential $\Phi \sim 1 / r^{* 3}$. The latter form, with different prefactors, has been found in previous studies on charged [50], thermophoretic [51,52], and active colloids $[38,53,54]$. Thus, near a liquid-solid boundary, the scaling of Fig. 2(b) is modified to $r^{5} \propto t$. Thus, the optofluidic mechanism described by the monopole has a wider applicability. We believe that monopolar flow rationalizes a great variety of phenomena in phoretic [55] and active matter [54] and that its relevance will be widely appreciated in due course.

The strength of the potential $\Phi$ when compared with the thermal energy $k_{B} T$ determines the onset of crystalline order. Denoting it by

$$
\Phi_{0}=\frac{1}{4 \pi \eta \mu_{\|}(1+\lambda)} \cdot \frac{\mu_{T}}{\mu_{\perp}} \cdot \partial_{z} T^{\infty}
$$

and using parameters $\mu_{T}=10 \mu \mathrm{m}^{2} \mathrm{~s}^{-1} \mathrm{~K}^{-1}, \lambda=30$, and $\eta_{w}=8.9 \times 10^{-4} \mathrm{Pas}$, we get $\Phi_{0} \sim 100 k_{B} T$ when $\partial_{z} T^{\infty}=$ $15 \mathrm{~K}_{\mu \mathrm{m}}^{-1}$. Here, we have used the experimentally measured positions in Fig. 2(c) to estimate the $\partial_{z} T^{\infty}$ from other known parameters. The strength is proportional to the thermal gradient and leads, curiously, to freezing by heating and melting by cooling. We show this explicitly in Fig. 3(a) by direct numerical simulations of Eq. (3) as a function of the strength of the optofluidic potential.

Short-range forces.-Once the long-ranged optofluidic interaction draws particles into the center of force, shortranged optical binding forces act to enhance crystalline order. The optical binding force is obtained from a numerical solution of the Maxwell equations in the Mie approximation (detailed in [24]). It is shorter in range than the optofluidic force but, being oscillatory and anisotropic [see Fig. 2(d)], couples to both positional and bond order of
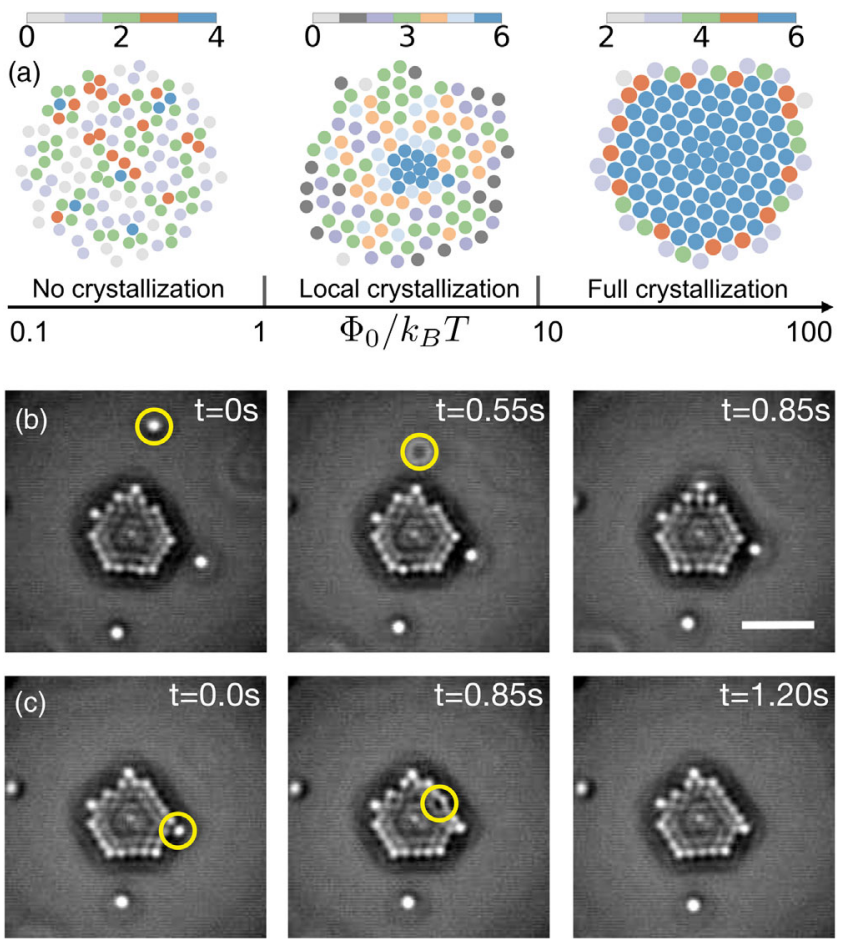

FIG. 3. (a) Competition between the optofluidic potential and Brownian motion determines the extent of crystalline order. Order is complete when the strength of the potential $\Phi_{0} / k_{B} T>10$, partial when $\Phi_{0} / k_{B} T \sim 1$, and absent when $\Phi_{0} / k_{B} T<1$. Particles are colored by their coordination number, which serves as a measure of order. (b) An untethered particle (yellow circle) is convected away from the interface by the optofluidic flow of Fig. 2(b). (c) Short-range forces promote the rapid annealing of a defect (yellow circle) produced by a particle colliding with the crystal (yellow circle) in the first frame. The scale bar is $10 \mu \mathrm{m}$.

the colloidal crystal. Its effect can be inferred indirectly from the rapid annealing of a defect (yellow circle) produced by the collision of a tethered particle with the crystallite, as shown in Fig. 3(c). We have not studied this coupling in detail and leave it to future work.

Conclusion.-Our experiments show how a novel nonequilibrium optofluidic force can be used to transport particles toward (or away) from an optically trapped "seed" particle. It is important to distinguish that the optofluidic force field is qualitatively different from the light-controlled thermoelectric fields generated in a medium, which contains a mixture of surfactant, ions, and micellar depletants [15]. It is also different from the thermo-osmotic flow generated by the differential heating of trapped Janus particles [56]. Theoretical analysis shows that the optofluidic force can be described in terms of the gradient of a potential, whose strength is proportional to the temperature gradient at the location of the seed. Untrapped particles couple to this potential regardless of their material properties, enabling the optofluidic manipulation of particles that cannot, otherwise, be optically trapped. Both the location of 
the potential and its strength can be modulated by the laser and its sign can be altered by changing the ratio of thermal conductivities of the liquids. We foresee this to lead to novel mechanisms of switchable, addressable transport in microfluidics, controlled self-assembly of active colloids and the metamaterial synthesis.

We thank Professor M. E. Cates, Professor D. Frenkel, and Professor E. J. Hinch for helpful discussions. A. C. thanks the ETN-COLLDENSE (H2020-MCSA-ITN-2014, Grant No. 642774) and the Winton Programme for the Physics of Sustainability. R. S. acknowledges the support of a Royal Society-SERB Newton International Fellowship. D. J. is thankful for support from the Udayan Care-VCare grant, the Nehru Trust for Cambridge University, the Schlumberger Foundation, Faculty for the Future Program, and Hughes Hall Santander Bursary Scholarship. R. A. thanks the Isaac Newton Trust for an Early Career Grant. Work was funded in part by the European Research Council under the EU's Horizon 2020 Program, Grant No. 740269.

*ee247@cam.ac.uk

[1] A. Ashkin, J. M. Dziedzic, J. E. Bjorkholm, and S. Chu, Opt. Lett. 11, 288 (1986); D. Baresch, J.-L. Thomas, and R. Marchiano, Phys. Rev. Lett. 116, 024301 (2016).

[2] K. Svoboda, C. F. Schmidt, B. J. Schnapp, and S. M. Block, Nature (London) 365, 721 (1993).

[3] J. Hofkens, J. Hotta, K. Sasaki, H. Masuhara, and K. Iwai, Langmuir 13, 414 (1997).

[4] J. E. Molloy, Science 300, 2045 (2003).

[5] M. Polin, D. G. Grier, and S. R. Quake, Phys. Rev. Lett. 96, 088101 (2006).

[6] D. Mizuno, C. Tardin, C. F. Schmidt, and F. C. MacKintosh, Science 315, 370 (2007).

[7] S. C. Chapin, V. Germain, and E. R. Dufresne, Opt. Express 14, 13095 (2006).

[8] T. Čiźmár, L. C. D. Romero, K. Dholakia, and D. L. Andrews, J. Phys. B 43, 102001 (2010).

[9] U. Tkalec, M. Ravnik, S. Čopar, S. Žumer, and I. Muševič, Science 333, 62 (2011).

[10] M. M. Burns, J.-M. Fournier, and J.A. Golovchenko, Science 249, 749 (1990).

[11] D. L. J. Vossen, M. A. Plaisier, and A. van Blaaderen, in Optical Trapping and Optical Micromanipulation, edited by K. Dholakia and G. C. Spalding (International Society for Optics and Photonics, 2004), p. 755.

[12] C. D. Mellor and C. D. Bain, Chem. Phys. Chem. 7, 329 (2006).

[13] A. H. Work and S. J. Williams, Soft Matter 11, 4266 (2015).

[14] T. Kudo, S. F. Wang, K. I. Yuyama, and H. Masuhara, Nano Lett. 16, 3058 (2016).

[15] L. Lin, J. Zhang, X. Peng, Z. Wu, A. C. H. Coughlan, Z. Mao, M. A. Bevan, and Y. Zheng, Sci. Adv. 3, e1700458 (2017).

[16] J. Liu and Z.-Y. Li, Photonics Res. 5, 201 (2017).

[17] K. Namura, K. Nakajima, K. Kimura, and M. Suzuki, Appl. Phys. Lett. 106, 043101 (2015).
[18] A. Karbalaei, R. Kumar, and H. J. Cho, Micromachines 7, 13 (2016).

[19] M.-T. Wei, J. Ng, C. T. Chan, and H. D. Ou-Yang, Sci. Rep. 6, 38883 (2016).

[20] G. Whitesides and B. Grzybowski, Science 295, 2418 (2002).

[21] D. Joshi, D. Bargteil, A. Caciagli, J. Burelbach, Z. Xing, A. S. Nunes, D. E. P. Pinto, N. A. M. Araújo, J. Brujic, and E. Eiser, Sci. Adv. 2, e1600881 (2016).

[22] B. J. Park and E. M. Furst, Langmuir 24, 13383 (2008).

[23] B. J. Park and E. M. Furst, Soft Matter 7, 7676 (2011).

[24] See Supplemental Material at http://link.aps.org/ supplemental/10.1103/PhysRevLett.125.068001 for the details of the calculations, experiments, numerics, and movies of crystallization, which includes Refs. [25-49].

[25] A. Caciagli, D. Joshi, J. Kotar, and E. Eiser, arXiv: 1703.08210 .

[26] T. Yanagishima, D. Frenkel, J. Kotar, and E. Eiser, J. Phys. Condens. Matter 23, 194118 (2011).

[27] L. Di Michele, T. Yanagishima, A. R. Brewer, J. Kotar, E. Eiser, and S. Fraden, Phys. Rev. Lett. 107, 136101 (2011).

[28] J. Gelles, B. J. Schnapp, and M. P. Sheetz, Nature (London) 331, 450 (1988); L. Vinet and A. Zhedanov, J. Phys. A 44, 085201 (2011).

[29] M. K. Cheezum, W. F. Walker, and W. H. Guilford, Biophys. J. 81, 2378 (2001).

[30] O. Otto, F. Czerwinski, J. L. Gornall, G. Stober, L. B. Oddershede, R. Seidel, and U. F. Keyser, Opt. Express 18, 22722 (2010).

[31] S. W. Hell, G. Reiner, C. Cremer, and E. H. K. Stelzer, J. Microsc. 169, 391 (1993).

[32] T. Brettschneider, G. G. Volpe, L. Helden, J. Wehr, and C. Bechinger, Phys. Rev. E 83, 041113 (2011).

[33] P. Jones, O. Maragó, and G. Volpe, Optical Tweezers: Principles and Applications (Cambridge University Press, Cambridge, England, 2015), ISBN 978-1-107-05116-4.

[34] S. Bera, S. Paul, R. Singh, D. Ghosh, A. Kundu, A. Banerjee, and R. Adhikari, Sci. Rep. 7, 41638 (2017).

[35] R. Singh, D. Ghosh, and R. Adhikari, Phys. Rev. E 98, 012136 (2018).

[36] J. Anderson, Annu. Rev. Fluid Mech. 21, 61 (1989).

[37] R. Singh and R. Adhikari, J. Phys. Commun. 2, 025025 (2018).

[38] R. Singh, R. Adhikari, and M. E. Cates, J. Chem. Phys. 151, 044901 (2019).

[39] K. Aderogba and J. Blake, Bulletin of the Australian Mathematical Society 19, 309 (1978).

[40] C. Pozrikidis, Boundary Integral and Singularity Methods for Linearized Viscous Flow (Cambridge University Press, 1992), pp. 1-259, ISBN 0-521-40693-5.

[41] R. Singh and R. Adhikari, J. Open Source Software 5, 2318 (2020).

[42] L. Mandel and E. Wolf, Optical Coherence and Quantum Optics (Cambridge University Press, Cambridge, 1995).

[43] E. Wolf, Proc. R. Soc. A 253, 349 (1959).

[44] B. Richards and E. Wolf, Proc. R. Soc. A 253, 358 (1959).

[45] A. Caciagli, Ph.D. thesis, University of Cambridge, 2020.

[46] J. Burelbach, M. Zupkauskas, R. Lamboll, Y. Lan, and E. Eiser, J. Chem. Phys. 147, 094906 (2017). 
[47] J. Burelbach, D. B. Brückner, D. Frenkel, and E. Eiser, Soft Matter 14, 7446 (2018).

[48] J. Burelbach, D. Frenkel, I. Pagonabarraga, and E. Eiser, Eur. Phys. J. E 41, 7 (2018).

[49] J. Wei, S. Ramírez-Hinestrosa, J. Dobnikar, and D. Frenkel, Soft Matter 16, 3621 (2020).

[50] T. M. Squires, J. Fluid Mech. 443, 403 (2001).

[51] R. Di Leonardo, F. Ianni, and G. Ruocco, Langmuir 25, 4247 (2009).

[52] F. M. Weinert and D. Braun, Phys. Rev. Lett. 101, 168301 (2008).
[53] R. Singh and R. Adhikari, Phys. Rev. Lett. 117, 228002 (2016).

[54] A. Bolitho, R. Singh, and R. Adhikari, Phys. Rev. Lett. 124, 088003 (2020).

[55] P. Wirnsberger, D. Fijan, R. A. Lightwood, A. Šarić, C. Dellago, and D. Frenkel, Proc. Natl. Acad. Sci. U.S. A. 114, 4911 (2017).

[56] S. M. Mousavi, I. Kasianiuk, D. Kasyanyuk, S. K. Velu, A. Callegari, L. Biancofiore, and G. Volpe, Soft Matter 15, 5748 (2019). 